Physics

Electricity \& Magnetism fields

\title{
Identification of electric charge distribution using dual reciprocity boundary element models
}

Yonghao Sun

Okayama University
Yukio Kagawa

Okayama University

This paper is posted at eScholarship@OUDIR : Okayama University Digital Information Repository.

http://escholarship.lib.okayama-u.ac.jp/electricity_and_magnetism/161 


\title{
Identification of Electric Charge Distribution Using Dual Reciprocity Boundary Element Models
}

\author{
Yonghao Sun and Yukio Kagawa \\ Department of Electrical and Electronic Engineering, Okayama University \\ Tsushima-naka 3-1-1, Okayama 700 Japan.
}

\begin{abstract}
Electric charges or sources distributed in space or field are identified from the data observed over the field boundary using dual reciprocity boundary element models. The technique makes it possible to identify a single charge directly. For modelling the field, the variational formulation is employed for which the regular boundary integral approach is incorporated to avoid the singularity.
\end{abstract}

\section{INTRODUCTION}

The boundary element analysis of the Poisson's equation requires the cell integration for the domain. The discretization of the interior domain means loss of its "boundary only" nature. A great deal of effort has gone into eliminating the domain integrals. The most promising approach to this is the use of Dual Reciprocity Method (DRM)[1].

The identification of the locations and magnitudes of the external forces in a domain using a boundary element algorithm has been proposed by many investigators[2 4]. The methods in most cases are based on minimizing the sum of the squares of the relative errors evaluated over the boundary.

We proposed a procedure to be called simulated charge searching approach [5] in which the DRM is extended to the problems of the identification of electric charge distribution. In the present work, the simulated charge searching approach is extended to identify both the location and magnitude of the electric charge without iterative calculation. The case when the charges are partially uniformly distributed is then examined in which the shape of the domain is to be determined by minimizing the sum of the squares of the relative errors evaluated over the boundary. The variational formulation is employed to form the boundary integral expression for which the regular boundary integral procedure[6] is incorporated to avoid the singular integrals.

\section{FORMULATION FOR THE DRM BOUNDARY ELEMENT METHOD}

Defining $\psi$ as the electric scalar potential, and $\rho$ as the space charge density distributed in domain $\Omega$, the functional of hybrid form corresponding to Poisson's equation can be expressed as

$$
\begin{array}{r}
\Pi(\psi, \tilde{\psi}, \tilde{p})=\frac{1}{2} \int_{\Omega}(\nabla \psi)^{2} d \Omega-\int_{\Gamma}(\psi-\tilde{\psi}) \tilde{p} d \Gamma \\
-\int_{\Gamma_{2}} \tilde{\psi} \hat{p} d \Gamma-\int_{\Omega} \frac{\rho}{\varepsilon} \psi d \Omega
\end{array}
$$

Manuscript received March 19, 1996. where $\tilde{\psi}, \tilde{p}(=\partial \tilde{\psi} / \partial n)$ are the potential and flux on boundary $\Gamma$, and $\hat{p}(=\partial \hat{\psi} / \partial n)$ is the forcing or exciting term prescribed on boundary $\Gamma_{2}$. The expression allows the discrepancy between potential $\psi$ closest to the boundary in the domain and potential $\tilde{\psi}$ on the boundary. $\varepsilon$ is the permittivity. Taking variation with respect to $\psi, \tilde{\psi}$ and $\tilde{p}$ respectively, we obtain the Poisson's equation or the governing equation

$$
\nabla^{2} \psi=-\frac{\rho}{\varepsilon}=b \quad \text { in } \Omega
$$

and the boundary conditions

$$
\left.\begin{array}{ll}
\psi=\tilde{\psi} & \text { on } \Gamma \\
\tilde{\psi}=\hat{\psi} & \text { on } \Gamma_{1} \\
p=\tilde{p} & \text { on } \Gamma \\
\tilde{p}=\hat{p} & \text { on } \Gamma_{2}
\end{array}\right\}
$$

The potential $\psi$ can be expressed as the sum of a particular solution $\varphi$ and a fundamental solution $\phi$, so that

$$
\psi(x, y)=\varphi(x, y)+\phi(x, y)
$$

Dividing the boundary into elements $\Gamma_{k}(k=1,2, \ldots M)$, and locating simulated charges $\alpha_{j}(j=1,2, \ldots, L)$ inside the domain, and fictitious charges $\beta_{k}(k=1,2, \ldots, M)$ outside the domain, the particular solution and fundamental solution at arbitrary point $i$ in the field is expressed in terms of a linear combination of the contributions from each source $\alpha_{j}$ and $\beta_{k}$, that is

$$
\begin{aligned}
\varphi_{i} & =\sum_{j=1}^{L} \varphi_{i j}^{*} \alpha_{j}=\left\{\varphi^{*}\right\}_{i}^{T}\{\alpha\} \\
\phi_{i} & =\sum_{k=1}^{M} \phi_{i k}^{*} \beta_{k}=\left\{\phi^{*}\right\}_{i}^{T}\{\beta\}
\end{aligned}
$$

where

$$
\begin{gathered}
\left\{\varphi^{*}\right\}_{i}=\left\{\varphi_{i 1}^{*}, \varphi_{i 2}^{*}, \ldots \ldots, \varphi_{i L}^{*}\right\},\left\{\phi^{*}\right\}_{i}=\left\{\phi_{i 1}^{*}, \phi_{i 2}^{*}, \ldots \ldots, \phi_{i M}^{*}\right\} \\
\{\alpha\}=\left\{\alpha_{1}, \alpha_{2}, \ldots \ldots, \alpha_{L}\right\},\{\beta\}=\left\{\beta_{1}, \beta_{2}, \ldots \ldots, \beta_{M}\right\}
\end{gathered}
$$

and $\varphi_{i j}^{*}$ is the particular solution of Poisson's equation $\nabla^{2} \varphi_{i j}^{*}=f_{i j}$, where $f_{i j}$ is an approximate function, the linear combination of which forms the forcing terms as one will see later. $\phi_{i k}^{*}$ is the fundamental solution of Laplace's equation whose value is evaluated at $i$ for a unit source given at point $k$ outside the domain.

By substituting equation (3) into (2), the Poisson's equation can be replaced by Laplace's equation

$$
\nabla^{2} \phi=0 \quad \text { in } \Omega
$$

and the expression over the boundary is given as 


$$
\tilde{\phi}(x, y)=\tilde{\psi}(x, y)-\tilde{\varphi}(x, y) \quad \text { on } \Gamma
$$

Defining $\phi$ in domain $\Omega$, and $\tilde{\phi}, \tilde{q}(=\partial \tilde{\phi} / \partial n)$ on boundary $\Gamma$, equation (4) can also be expressed as the functional of hybrid form

$$
\begin{array}{r}
\Pi(\phi, \tilde{\phi}, \tilde{q})=\frac{1}{2} \int_{\Omega} \varepsilon(\nabla \phi)^{2} d \Omega-\int_{\Gamma} \varepsilon(\phi-\tilde{\phi}) \tilde{q} d \Gamma \\
-\int_{\Gamma_{2}} \varepsilon \tilde{\phi} \hat{q} d \Gamma
\end{array}
$$

where $\hat{q}$ is the forcing term on the boundary $\Gamma_{2}$.

Integrating by parts the first term in equation (6) to eliminate the domain integration results in the expression with boundary integral only

$$
\begin{array}{r}
\Pi(\phi, \tilde{\phi}, q, \tilde{q})=\frac{1}{2} \int_{\Gamma} \varepsilon \phi q d \Gamma-\int_{\Gamma} \varepsilon(\phi-\tilde{\phi}) \tilde{q} d \Gamma \\
-\int_{\Gamma_{2}} \varepsilon \tilde{\phi} \hat{q} d \Gamma
\end{array}
$$

The variation of the boundary integral expression (7) results in

$$
[K]\{\tilde{\phi}\}-[G]\left\{\frac{\partial \tilde{\phi}}{\partial n}\right\}=\{0\}
$$

in discretized form[7]. Applying the relation (5) into equation (8), one can write

$$
\begin{aligned}
& {[K][\{\tilde{\psi}\}-\{\tilde{\varphi}\}]-[G]\left[\{\tilde{p}\}-\left\{\frac{\partial \tilde{\varphi}}{\partial n}\right\}\right]=} \\
& {[K]\left[\{\tilde{\psi}\}-\left\{\sum_{j=1}^{L} \varphi_{i j}^{*} \alpha_{j}\right\}\right]-[G]\left[\{\tilde{p}\}-\left\{\sum_{j=1}^{L} \frac{\partial \varphi_{i j}^{*}}{\partial n} \alpha_{j}\right\}\right]=\{0\}(9)}
\end{aligned}
$$

Finally, the discretized system equation becomes

$$
[K]\{\tilde{\psi}\}-[G]\{\tilde{p}\}=\{[K][H]-[G][Q]\}\{\alpha\}=[S]\{\alpha\}
$$

where

$$
[S]=[K][H]-[G][Q]
$$

The components of the matrix $[G],[H]$ and $[Q]$ are

and

$$
G_{k i}=\int_{\Gamma_{i}} \phi_{k i}^{*} d \Gamma_{i}, H_{i j}=\varphi_{i j}^{*}, Q_{i j}=\frac{\partial \varphi_{i j}^{*}}{\partial n}=q_{i j}^{*}
$$

$$
[K]=[E][R]
$$

in which the component of $[E]$ is

$$
\begin{aligned}
& E_{k i}=\frac{1}{2} \sum_{m=1}^{M} \int_{\Gamma_{m}} \phi_{k m}^{*} \frac{\partial \phi_{i m}^{*}}{\partial n} d \Gamma_{m}+\frac{1}{2} \sum_{m=1}^{M} \int_{\Gamma_{m}} \phi_{i m}^{*} \frac{\partial \phi_{k m}^{*}}{\partial n} d \Gamma_{m} \\
& \text { and } \\
& {[R]=\left([G]^{-1}\right)^{T}[U] \quad(k, i=1,2, \ldots, M, j=1,2, \ldots, L)}
\end{aligned}
$$

$[U]$ is a diagonal matrix and the component of $[U]$ corresponds to the length of element $\Gamma_{i}$ over the boundary $\Gamma$ as in the present formulation where the constant elements are used. Equation (10) is the dual reciprocity expression for which regular boundary element approach is incorporated to avoid the singular integrals by locating fictitious charges $\beta_{k}$ outside the domain.

The DRM implies an expansion for $b(\mathrm{x}, \mathrm{y})$ of the type

$$
b_{i}=\sum_{j=1}^{L} f_{i j} \alpha_{j}=\sum_{j=1}^{L}\left(\nabla^{2} \varphi_{i j}^{*}\right) \alpha_{j}
$$

$L$ is the number of the node of simulated charges $\alpha_{j}$, and $\varphi_{i j}^{*}$ is the particular solution of Poisson-type equation

$$
\nabla^{2} \varphi_{i j}^{*}=f_{i j}
$$

where $f_{i j}$ is an approximate expansion function for $b$. The coefficient can be expressed in a matrix form as

$$
\{\alpha\}=[f]^{-1}\{b\}
$$

where

$$
\{b\}=\left\{b_{1}, b_{2}, \ldots \ldots, b_{L}\right\}, \quad b_{i}=b\left(x_{i}, y_{i}\right)
$$

Equation (12) is substituted into equation (10) resulting in

$$
[K]\{\tilde{\psi}\}-[G]\{\tilde{p}\}=[S][f]^{-1}\{b\}
$$

In the forward analysis, $b(x, y)$ is known, so that equation (13) arrives at the conventional boundary element expression.

\section{A. Inversion}

\section{Single ChaRge IDENTIFICATION}

The inverse problem shown in Fig.1 is considered.

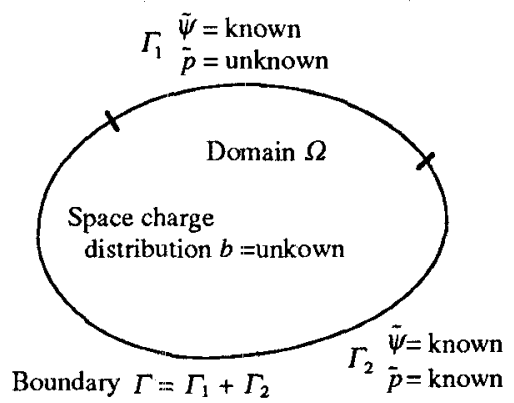

Fig.1. Field and boundary conditions for inverse problem.

The source term $b$ is a function of $(x, y)$ in domain $\Omega$, bounded by $\Gamma\left(=\Gamma_{1}+\Gamma_{2}\right)$. The potential $\tilde{\psi}$ and flux $\tilde{p}$ on $\Gamma_{2}$, and $\tilde{\psi}$ on $\Gamma_{1}$ are known or observed. We try to find the value $\tilde{p}$ on boundary $\Gamma_{1}$ and the source distribution $b(x, y)$. We first allocate coefficients $\alpha$, and then calculate the values of $\alpha$, whose linear combination gives the approximate values for $b(x, y)$ with equation (11). Boundary $\Gamma_{1}$ is divided into $k$ elements, and $\Gamma_{2}$ is divided into $M-k$ elements. Reordering the equations in such a way that all the unknown, coefficients $\alpha$ and fluxes $\tilde{p}$ on $\Gamma_{1}$, assigning that the matrix on the left is $[A]$, the unknown vector is $\{X\}$, are placed on the left hand side, and the known values $\{\hat{W}\}$ on the right hand side. One can write equation (13) as

$$
[A]\{X\}=\{\hat{W}\}
$$

If the sum of the number of the known, $\tilde{\psi}$ and $\tilde{p}$ on $\Gamma_{2}$, and on $\Gamma_{1}$, is greater than the number of the unknown $\alpha$ and $\tilde{p}$ on $\Gamma_{1}$, then the order of equation (14) is greater than the number of the unknown variables. The equation can be solved for $\{\alpha\}$ by usual least-squares method and the source distribution $b(x, y)$ can readily be obtained by equation (11). 
Now we consider the case when a positive source charge situated in the domain. Here we propose a procedure which could be called a simulated charge searching approach. Since term $b(x, y)$ is expanded as the linear combination of simulated charges, that is

$$
b_{i} \approx \sum_{j}^{L} \alpha_{j} \delta\left(x_{i}-x_{j}, y_{i}-y_{j}\right)
$$

wherc $\delta\left(x_{i}-x_{j}, y_{i}-y_{j}\right)$ is a delta function, which implies the approximate function

$$
f_{i j}=\delta\left(x_{i}-x_{j}, y_{i}-y_{j}\right)=\delta_{i j}
$$

The particular solution for $\nabla^{2} \varphi_{i j}^{*}=\delta_{i j}$ is therefore

$$
\varphi_{i j}^{*}=-\frac{1}{2 \pi}\left(\ln r_{i j}\right)
$$

$r_{i j}$ is the distance from point $i$ in the domain to the boundary node $j$ where the simulated charges are allocated.

Allocating $L$ number of simulated charges at locations $\left(x_{j}\right.$, $y_{j}$ ), we can calculate the magnitudes $\alpha_{j}$ by solving equation (14). We estimate the best possible positions $\left(x_{c}, y_{c}\right)$ of the charge by averaging the simulated charges in such a way that

$$
x_{c}=\sum_{j}^{L} \alpha_{j} x_{j} / \sum_{j}^{L} \alpha_{j}, y_{c}=\sum_{j}^{L} \alpha_{j} y_{j} / \sum_{j}^{L} \alpha_{j}
$$

and the best possible magnitude $\alpha_{c}$ by summing total charges up as

$$
\alpha_{c}=\sum_{j}^{L} \alpha_{j}
$$

\section{B. Numerical Examples}

A closed square domain, $\mathrm{x}=0.0 \sim 1.0$ by $\mathrm{y}=0.0 \sim 1.0$, is considered in which a single charge of magnitude 1.0 is placed at location $\left(x_{c}, y_{c}\right)=(0.4,0.3)$. We assume that the values of $\tilde{\psi}$ and $\tilde{p}$ are both observed or measured on boundary $\Gamma$, for which we utilize the forward solution in the present simulation. The boundary is divided into 32 constant elements.

In the inverse analysis, the boundary is again divided into 32 constant elements for which the same number of the fictitious charges are allocated outside the domain along the boundary and 9 simulated charges are allocated within the region. As the fictitious charges are arranged outside the domain, all of the boundary integrals do not involve singularity, which can easily be evaluated.

The position of the charge is estimated to be at $x_{c}=0.3977$, $y_{c}=0.2978$ and the magnitude to be $\alpha_{c}=1.0003$ with the help of equation (15) and (16), which is very close to magnitude 1.0 at location $(0.4,0.3)$ originally set.

Next, the case when the values of $\tilde{\psi}$ and $\tilde{p}$ are both specified or observed on boundary $\Gamma_{2}$ but the value of $\tilde{\psi}$ is only known on $\Gamma_{1}$. The magnitude and position of a single charge are to be identified together with flux $\tilde{p}$ on $\Gamma_{1}$. Boundary $\Gamma_{2}$ is divided into 14 and boundary $\Gamma_{1}$ into 4 constant elements. A charge of magnitude 1.0 is again placed at location $(0.4,0.3)$. In inversion, the magnitude and the position obtained are $\alpha_{c}=1.0246, x_{c}=0.4306$ and $y_{c}=0.2920$.

\section{UNIFORMLY DISTRIBUTED CHARGES OF ARBITRARY SHAPE}

\section{A. Distributed Charges in a Circle}

One can extend the discussion further to the case when the charges are uniformly distributed in a circle in the square field $(6 \times 6)$ in which the boundary conditions are prescribed on the domain boundary. The center of the circle is at $\left(x_{c}, y_{c}\right)=(2.0$, 2.0 ), the radius is $R=0.8$ and the charge density is known, $\rho$ $=100$. Center of the circle and its radius are to be determined from the data observed on the boundary.

By solving equation (14), the circle's center is determined as $\left(x_{c}, y_{c}\right)=(1.9874,1.9872)$ and the total charge is $\alpha_{c}=198.2527$. As the density is known, equivalent radius $R$ can be calculated from $\alpha_{c}=\pi R^{2} \rho$.

If uniformly distributed charges are of an arbitrary shape, the inversion process is not so simple and must be made in successive manner. We first calculate the central position of the distributed charges and the magnitude of the total charges by using the simulated charge searching approach proposed, and then find the equivalent radius as demonstrated above. Taking this as the initial boundary shape, true shape must be searched by shifting the boundary so as to minimize the error function or objective function evaluated over the boundary.

\section{B. Formulation}

If the charges are uniformly distributed in the domain $\Omega^{B}$ within field $\Omega^{A}$ shown in Fig.2, the term $b(\mathrm{x}, \mathrm{y})$ of the Poisson's equation is constant $b_{0}$ and equation (11) can be expressed as

$$
\sum_{j=1}^{L} f_{i j} \alpha_{j}=\alpha_{k}=b_{0} \quad \text { in } \Omega^{B}
$$

Only one simulated charge could be used, that is, $\alpha_{j}$ s are entirely zeros except at position $k$ where $\alpha_{k}=b_{0}$. Here we allocate the simulated charge $\alpha_{k}$ in the center of domain $\Omega^{B}$. With function $f_{i k}=1$ adopted, the particular solution $\varphi_{i k}^{*}$ is

$$
\varphi_{i k}^{*}=\frac{r_{i k}^{2}}{4}, r_{i k}=\sqrt{\left(x_{i}-x_{k}\right)^{2}+\left(y_{i}-y_{k}\right)^{2}}
$$

In this case, equation $(10)$ becomes

$$
[K][\tilde{\psi}\}-[G]\{\tilde{p}\}=\left[[K]\left\{\varphi^{*}\right\}-[G]\left\{q^{*}\right\}\right] b_{0}
$$

In region $\Omega^{A}$, since there is no charge, the governing equation is

$$
\nabla^{2} \tilde{\psi}^{A}=0
$$

and in region $\Omega^{B}$ in which the charges are uniformly distributed, it is

$$
\nabla^{2} \tilde{\psi}^{B}=-\frac{\rho}{\varepsilon}=b_{0}
$$

The boundary $\Gamma_{A}=\Gamma_{O}+\Gamma_{I}$ of domain $\Omega^{A}$ is divided into $M_{O}+M_{I}$ elements and the boundary $\Gamma_{B}=\Gamma_{l}$ of domain $\Omega^{B}$ is divided into $M_{I}$ elements. The discretized boundary element expressions can be expressed for each region as 


$$
\begin{gathered}
{\left[\left[K_{O}^{A}\right]\left[K_{I}^{A}\right]\right]\left[\begin{array}{c}
\left\{\tilde{\psi}_{O}^{A}\right\} \\
\left\{\tilde{\psi}_{I}^{A}\right\}
\end{array}\right]=\left[\left[G_{O}^{A}\right]\left[G_{I}^{A}\right]\right]\left[\begin{array}{c}
\left\{\tilde{p}_{O}^{A}\right\} \\
\left\{\tilde{p}_{I}^{A}\right\}
\end{array}\right]} \\
{\left[K_{I}^{B}\right]\left\{\tilde{\psi}_{I}^{B}\right\}-\left[G_{I}^{B}\right]\left\{\tilde{p}_{I}^{B}\right\}=\left\{d^{B}\right\}}
\end{gathered}
$$

where

$$
\left\{d^{B}\right\}=\left[\left[K_{I}^{B}\right]\left\{\varphi^{*}\right\}-\left[G_{I}^{B}\right]\left\{q^{*}\right\}\right] b_{0}
$$

Superscript $A$ or $B$ indicates the boundary of each domain, and $I$ indicates the interface boundary. Along the interface, both $\psi$ and $p$ are unknown but compatible, so that equilibrium conditions are

$$
\begin{gathered}
\tilde{\psi}_{I}^{A}=\tilde{\psi}_{I}^{B}=\tilde{\psi}_{I} \\
\tilde{p}_{I}^{B}=-\tilde{p}_{I}^{A}=-\tilde{p}_{I}
\end{gathered}
$$

Equations (19) and (20) are now combined to form

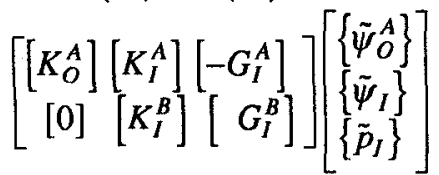

$$
\begin{aligned}
& -\left[\begin{array}{cc}
{\left[G_{O}^{A}\right][0]} \\
{[0]} & {[0]}
\end{array}\right]\left[\begin{array}{c}
\left\{\tilde{p}_{O}^{A}\right\} \\
\{0\}
\end{array}\right]=\left[\begin{array}{c}
\{0\} \\
\left\{d^{B}\right\}
\end{array}\right]
\end{aligned}
$$

Equation (23) can be rearranged to provide the known variables on the right-hand side and to obtain the same form as equation (14).

\section{A Numerical Example}

The task of the inverse problem considered here is to determine the position of boundary $\Gamma_{I}$ of rectangular domain $\Omega^{B}$ in which the charges are uniformly distributed as shown in Fig.2. In the inverse analysis, first, by using equation (14) we obtained the equivalent circular radius, which is used as the initial boundary shape. The circumference is divided into 16 elements with radius $\left(R_{i}, i=1,2, \ldots, 16\right)$.

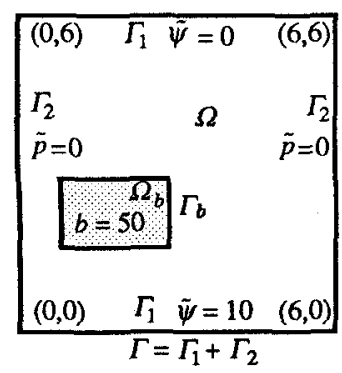

Fig. 2. Uniformly distributed charges and boundary conditions

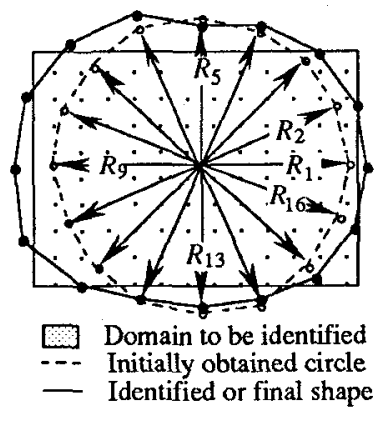

Fig.3. Inversion
The unknown interface boundary can be determined by varying the radial length $R_{i}$ to minimize the objective function

$$
E=\sum_{n=1}^{M_{0}}(\psi-\hat{\psi})^{2}
$$

which is chosen as a square sum of residuals between the measured potential $\hat{\psi} s$ at selected nodes on the boundary $\Gamma_{O}$ and the corresponding value $\psi \mathrm{s}$ computed by the equation (23). Davidon-Fletcher--Powell (DFP) method[8] is employed for minimizing the objective function. After 16 iterative computation, the determined shape is shown in Fig.3. The rectangular shape is not properly reconstructed. This is expected as the resolution would be within the length of the elements chosen on which the variables are assumed to be constant.

\section{CONCLUding REMARKS}

The use of DRM boundary element models was proposed to identify the distribution of the electric charges. The method is incorporated with a simulated charge approach to avoid the singular integral. In the case when a single charge is located in the domain, the position and magnitude are well identified by the direct inversion. This method could be used to give the first guess of the shape for the problem for which the arbitrary shape of the domain of uniformly distributed charges is to be determined. For the last case, the usual iterative method is inevitable.

\section{REFERENCES}

[1] P.W. Partridge, C.A. Brebbia and L.C. Wrobel, The Dual Reciprocity Boundary Element Method, Computational Mechanics Publications, Southampton, 1992.

[2] K. Ohnaka and K. Uosaki, "Identification of the external input of distributed-coefficient systems by the boundary element approach", Int. J. Control, vol. 43-4, pp.1125$1133,1986$.

[3] K. Ohnaka and K. Uosaki, "Boundary element approach for identification of point forces of distributed coefficient systems", Int. J. Control, vol. 49-1, pp.119127, 1989.

[4] S. Kubo, K. Ohnaka and K. Ohji, "Identification of heatsource and force using boundary element integrals", J. Japan Soc. Mech. Eng., vol. 54-A, pp.1329-1334, 1987.

[5] Y. Kagawa, Y. Sun and O. Matsumoto, "Inverse solution of Poisson equation using DRM boundary element model - Identification of space charge distribution", Inverse Problems Eng., vol. 1-2, pp.247-265, 1995.

[6] Y. Sun and Y. Kagawa, "Regular boundary integral solution with dual and complementary variational formulations applied to two-dimensional Laplace problems", Int. J. Numer. Modelling Electro. Networks, Devices, Fields, vol. 8-2, pp.127-137, 1995.

[7] Y. Sun and Y. Kagawa, "A variational approach to boundary elements - Two-dimensional Laplace problem", Int. J. Numer. Modelling Electro. Networks, Devices, Fields, vol. 7-1, pp.1-14, 1994.

[8] S.L.S. Jacoby, J.S. Kowalik and J.T. Pizzo, Iterative Methods for Nonlinear Optimization Problems, PrenticeHall, Englewood Cliffs, 1972. 